\title{
IMPLEMENTACIÓN DE PRÁCTICAS DE GOBIERNO CORPORATIVO EN COLOMBIA: UN ANÁLISIS DESDE EL ISOMORFISMO INSTITUCIONAL EN EMPRESAS FAMILIARES Y NO FAMILIARES*
}

\author{
DIÓGENES LAGOS CORTÉS**; JOSE BERNARDO BETANCOURT RAMÍREZ**** \\ \& GONZALO GÓMEZ-BETANCOURT***** \\ UNIVERSIDAD DE LA SABANA - LEGACY AND MANAGEMENT CONSULTING GROUP
}

Recibido/ Received/ Recebido: 13/04/2016 - Aceptado/ Accepted / Aprovado: 30/09/2016

\begin{abstract}
Resumen
Desde la perspectiva del isomorfismo institucional fue estudiada la implementación de prácticas de gobierno corporativo durante los años 2008 a 2014 en 105 empresas inscritas en el Registro Nacional de Valores y Emisores (RNVE) de Colombia. No se encontró evidencia para afirmar que en dicha implementación exista isomorfismo. Estos resultados pueden deberse al poco tiempo que tiene de promulgado el Código País (siete años), siendo un periodo de estudio corto para reflejar cambios significativos en la variación del índice de Gobierno Corporativo Código País (IGCCP) en Colombia. Se validó que la tendencia hacia la homogeneización en la implementación de prácticas de gobierno corporativo es diferente en las empresas familiares frente a las no familiares.

Palabras clave: Gobierno corporativo; Prácticas; Empresa familiar; Isomorfismo; Colombia.
\end{abstract}

Artículo de investigación enmarcado en la línea "Gobierno corporativo" del grupo de investigación Empresa Familiar de INALDE Business School, Universidad de La Sabana.

* Candidato a Doctor en Ciencias de la Administración, Universidad Nacional de La Plata (Argentina). Magister en Ingeniería Industrial, Universidad Industrial de Santander. Administrador de Empresas, Universidad Nacional de Colombia. Investigador del grupo Empresa Familiar, INALDE Business School, Universidad de La Sabana. Dirección Postal: Km. 7 Autopista Norte Costado Occidental, Chía (Colombia). Teléfono: (057-1) 8614444 Ext. 16043. Correo electrónico: diogenes.lagos@inalde.edu.co

*** Candidato a Doctor en Administración Estratégica de Empresas, Centrum Graduate Business School, Pontificia Universidad Católica (Perú). DBA (c) en Maastricht School of Management (Holanda). MBA, Universidad Internacional Iberoamericana (Puerto Rico). MDEEF, Universidad de León (España). Administrador de Instituciones de Servicio, Universidad de La Sabana. Miembro del grupo de investigación Empresa Familiar, INALDE Business School, Universidad de La Sabana. Docente de catedra en Posgrados, Facultad de Ciencias Administrativas y Contables, Universidad de La Salle. Director Administrativo, lymgroup.com (Colombia). Correo electrónico: jose.betancourt@inalde.edu.co

Ph.D. in Management, Universidad de Navarra (España). MBA, Inalde Business School. Ingeniero Industrial, Pontificia Universidad Javeriana. CEO Legacy y Management Consulting Group. Correo electrónico: gonzalo.gomez@lymgroup.com 


\title{
IMPLEMENTATION OF CORPORATE GOVERNANCE PRACTICES IN COLOMBIA: AN ANALYSIS FROM THE INSTITUTIONAL ISOMORPHISM IN FAMILY AND NONFAMILY COMPANIES
}

\begin{abstract}
From the perspective of institutional isomorphism, the adoption of corporate governance practices during the years 2008 to 2014 was studied in 105 companies registered in the Colombia's National Registry of Securities and Issuers (RNVE). No evidence was found to claim that there is isomorphism in this adoption. These results may be due to the short time that the Country Code has been enacted (seven years). Being a short study period to reflect significant changes in the variation of the Corporate Governance Index Country Code (IGCCP) in Colombia. It was validated that the tendency toward homogenization in the adoption of corporate governance practices is different in family versus non-family businesses.

Keywords: Corporate governance; practices; Family business; Isomorphism; Colombia.

\section{IMPLEMENTAÇÃO DE PRÁTICAS DE GOVERNO CORPORATIVO NA COLÔMBIA: UMA ANÁLISE DESDE O ISOMORFISMO INSTITUCIONAL EM EMPRESAS FAMILIARES E NÃO FAMILIARES}

\begin{abstract}
Resumo
A partir da perspectiva do isomorfismo institucional foi estudada a implementação de práticas de governo corporativo durante os anos 2008 a 2014 em 105 empresas inscritas no Registro Nacional de Valores e Emissores (RNVE) da Colômbia. Não se encontrou evidência para afirmar que em dita implementação exista isomorfismo. Estes resultados podem ser devido ao pouco tempo que tem de promulgado o Código País (sete anos), sendo um período de estudo curto para refletir mudanças significativas na variação do índice de Governo Corporativo Código País (IGCCP) na Colômbia. Validou-se que a tendência para a homogeneização na implementação de práticas de governo corporativo é diferente nas empresas familiares frente às não familiares.
\end{abstract}

Palavras chave: Governo corporativo; Práticas; Empresa familiar; Isomorfismo; Colômbia.

Lagos, D., Betancourt, J. \& Gómez-Betancourt, G. (2017) Implementación de prácticas de gobierno corporativo en Colombia: un análisis desde el isomorfismo institucional en empresas familiares y no familiares. En: Revista de la Facultad de Ciencias Económica: Investigación y Reflexión. rev.fac.cienc.econ, XXV (2), DOI: https://doi.org/10.18359/rfce.1832

JEL: D23, G30. 


\section{Introducción}

Las crisis financieras y los grandes escándalos empresariales han sido determinantes en la mayor atención que ha recibido el gobierno corporativo (Claessens, 2006). El entorno colombiano no ha sido ajeno a esta dinámica, en 2007 entró en vigencia el Código País ${ }^{1}$ para emisores de valores, posteriormente, en 2009 se creó la Guía Colombiana de Gobierno Corporativo para Sociedades Cerradas y de Familia. Desde entonces, muchas empresas colombianas se han comprometido con la implementación de buenas prácticas de gobierno ${ }^{2}$.

Diversos autores han estudiado el gobierno corporativo, principalmente desde las diferencias existentes, sin embargo, Powell \& Dimaggio (1991) plantearon un enfoque diferencial al preguntarse ¿por qué hay una homogeneidad tan sorprendente de formas y prácticas organizacionales? Desde esta perspectiva, intentaron explicar la homogeneidad y no la variación en las organizaciones. Powell \& Dimaggio muestran que en etapas iniciales los campos organizacionales ${ }^{3}$ presentan una diversidad de enfoques y formas, y a medida que el campo se va consolidando tienden hacia la homogeneización. Usando este enfoque, y considerando el gobierno corporativo como una innovación organizacional en empresas familiares (EF) y no familiares (ENF), se pretende identificar si en la implementación de las prácticas de gobierno corporativo definidas en el Código País se presenta isomorfismo. Para ello, se plantearon las siguientes preguntas: ¿la imple-

1 El Código País define 41 prácticas de gobierno corporativo de implementación voluntaria, y para su evaluación se utiliza la Encuesta Código País, que a través de 80 preguntas suministra información sobre el cumplimiento de dichas prácticas.

2 Según cifras de la Superintendencia Financiera de Colombia (SFC), a enero de 2014, 140 empresas inscritas en el Registro Nacional de Valores y Emisores (RNVE) habían implementado códigos de gobierno corporativo. No se tienen cifras acerca de cuantas sociedades cerradas y de familia han implementado códigos de gobierno corporativo.

3 El campo organizacional son aquellas organizaciones que, en conjunto, constituyen un área reconocida de la vida institucional: los proveedores principales, los consumidores de recursos y productos, las agencias reguladoras, y otras organizaciones que dan servicios o productos similares (Powell \& Dimaggio, 1991, p. 106). mentación de dichas prácticas tiende hacia la homogeneización?, ¿la regulación conlleva a similitudes en dichas prácticas?, ¿las empresas imitan a otras en la implementación de prácticas de gobierno corporativo?

Para responder a estas preguntas, en el presente estudio se usaron técnicas estadísticas para identificar la tendencia de las empresas frente a la implementación de prácticas medidas con el Índice de Gobierno Corporativo Código País (IGCCP) y así comprobar si existen comportamientos isomorfos. Las empresas objeto de análisis fueron clasificadas como EF y ENF, Emisores y No Emisores de bonos y acciones, y como Exitosas o No Exitosas en término de ventas. Mediante el análisis del coeficiente de variación y la varianza, se buscó evidencia estadística para identificar comportamientos isomorfos en la implementación de prácticas del IGCCP, y así validar si las empresas en Colombia cada vez conceden mayor importancia a las prácticas de gobierno corporativo definidas en el código país o sólo se adaptan a las tendencias del entorno.

El resto del documento se estructura como sigue: en la segunda parte se presenta la revisión de literatura y se realiza la formulación de las hipótesis. La metodología de investigación es presentada en la tercera parte. En la cuarta parte se presentan los resultados y se discute acerca de ellos. Finalmente, las conclusiones y limitaciones son presentadas en la quinta parte.

\section{Isomorfismo organizacional y gobier- no corporativo}

La teoría organizacional presenta a las organizaciones como diversas y diferenciadas, pero Powell \& Dimaggio (1991) desde una perspectiva diferente se preguntaron ¿por qué se presentaba una homogeneidad tan sorprendente de formas y prácticas organizacionales? Su objetivo era explicar la homogeneidad y no la variación en las organizaciones. Esta homogeneización conocida como isomorfismo, sugiere que a través de un proceso limitador se obliga a una organización en determinada población a parecerse a otras organizaciones que enfrentan las mismas condiciones ambientales (Powell 
\& Dimaggio, 1991). En los últimos 10 años, el gobierno corporativo en Colombia ha recibido mayor importancia, aunque son diversos los factores que han incidido en el auge de la implementación de buenas prácticas de gobierno, existe consenso en la literatura acerca de dos circunstancias que han potencializado dicha situación: i) la proliferación de las crisis financieras, y ii) los grandes escándalos empresariales (Claessens, 2006).

Desde la entrada en vigencia del Código País y de la Guía Colombiana de Gobierno Corporativo para Sociedades Cerradas y de Familia, muchas empresas en Colombia se han comprometido con la implementación de buenas prácticas. No obstante, pocas investigaciones se centran en comprender el proceso de implementación de dichas prácticas, y en términos generales se acepta que lo hacen por los múltiples beneficios asociados a su implementación (menor costo de capital, mayor rendimiento de las acciones, mayor eficiencia, un trato más favorable de todas las partes interesadas, etc.), siendo beneficiosa esta implementación para las empresas, los mercados, y los países (Claessens, 2006).

A pesar de los beneficios del buen gobierno corporativo, es posible que las empresas adopten dichas prácticas mediante comportamientos isomorfos que poco tienen que ver con mejoras en el desempeño. De acuerdo con Meyer \& Rowan (1977) el isomorfismo tiene algunas consecuencias cruciales para las organizaciones: i) incorpora elementos que son legitimados externamente, en lugar de seguir los términos de eficiencia; ii) emplean criterios de evaluación externos o ceremoniales para definir el valor de los elementos estructurales; y iii) reduce la turbulencia y mantiene la estabilidad por la dependencia de instituciones externas.

Powell \& Dimaggio (1991) identificaron tres mecanismos a través de los cuales sucede el cambio institucional isomorfo: i) isomorfismo coercitivo, que se debe a influencias políticas y al problema de legitimidad, este resulta de presiones tanto formales como informales que sobre unas organizaciones ejercen otras de las que dependen; ii) isomorfismo mimético, resulta de las respuestas estándares a la incertidumbre; e iii) isomorfismo normativo, asociado con la profesionalización.

Gunathilake \& Palli (2010) usaron la teoría del cambio institucional isomorfo para explicar por qué las organizaciones aceptan códigos voluntarios sobre gobierno corporativo y por qué dicha aceptación es un proceso que se desarrolla de forma rápida entre las organizaciones. Varios estudios han intentado validar estas proposiciones desde un punto de vista empírico, por ejemplo, Thorsell \& Cornelius (2009) encontraron que el proceso de selección de miembros de junta directiva en las empresas que ingresan a cotizar en la bolsa de valores de Suecia sigue comportamientos isomorfos de tipo coercitivo procedentes de los mecanismos de regulación existentes en el mercado.

Zakaria (2012) sugiere que el proceso de fijación de la remuneración de los ejecutivos en empresas de Reino Unido sigue comportamientos isomorfos. Dias Filho \& Moura (2013) encuentran que los bancos más pequeños tienden a copiar las prácticas de divulgación aprobadas por los más grandes. Moura, Magalhães, Aquino, \& Santos (2014) estudian la divulgación de prácticas de gobierno corporativo en el sector bancario en Brasil en comparación con las empresas que cotizan en la Bolsa de Valores de New York y encuentran que no existe evidencia que sugiera una tendencia hacia el isomorfismo.

Riaz, Ray, Ray, \& Kumar (2015) estudiaron las políticas de divulgación de la remuneración a nivel directivo y de la alta dirección en empresas multinacionales que operan en el mercado australiano y encontraron que estas empresas, en comparación con las empresas locales, son menos sensibles a mejorar los requisitos de divulgación, esto a menos que tengan una fuerte dependencia de recursos locales.

Con base en estos estudios se plantea la siguiente hipótesis:

$H_{1 a}$ : Cuanto mayor es el tiempo transcurrido desde la publicación del código de gobierno corporativo en Colombia (Código País), mayor es la tendencia hacia la homogeneización en la imple- 
mentación de dichas prácticas en las empresas inscritas en RNVE 4 .

Las EF presentan elementos en la estructura de propiedad, el ejercicio del poder y la dirección del negocio que son diferentes de las ENF (Maseda, Arosa $\&$ Iturralde, 2015). En la estructura de gobierno corporativo de una EF interactúan tres elementos: familia, gobierno y empresa (Suess, 2014), esta interacción hace que las EF tengan una dinámica diferente en la implementación de prácticas de gobierno corporativo. Por ejemplo, San Martin-Reyna y Duran-Encalada (2012) muestran que existen diferenciales sustanciales en las estructuras de gobierno corporativo entre las EF y las ENF. Esto sugiere que los procesos de homogeneización pueden ser diferentes en EF y ENF, entonces se busca validar la siguiente hipótesis:

$H_{1 b}$ : La tendencia hacia la homogeneización en la implementación de prácticas de gobierno corporativo es diferente en $E F$ y ENF.

De acuerdo con Meyer \& Rowan (1977) el cambio organizacional isomorfo de tipo coercitivo hace que las organizaciones que incorporan elementos racionalizados y legitimados socialmente en sus estructuras formales, maximicen su legitimidad y aumenten los recursos y posibilidades de supervivencia. Meyer \& Rowan (1977) sugieren que, aunque de forma general, las primeras organizaciones que adoptan innovaciones lo hacen con el objetivo de mejorar el desempeño, a medida que se difunde la innovación, se presenta que la implementación proporciona legitimidad, en lugar de mejorar el desempeño. Adicional se tiene que el hecho mismo de que se aprueban a través de alguna normatividad aumenta la probabilidad de su implementación

$4 \quad$ El RNVE tiene por objeto inscribir las clases y tipos de valores, así como los emisores de los mismos y las emisiones que estos efectúen, y certificar lo relacionado con la inscripción de dichos emisores clases y tipos de valores. La inscripción en este registro es requisito para aquellas entidades que deseen realizar una oferta pública de sus valores o que los mismos se negocien en un sistema de negociación.
(Powell \& Dimaggio, 1991). En el caso colombiano, el Código País está construido bajo el principio "cumpla o explique", de esta forma, cada empresa dependiendo de su situación particular decide libremente cuáles aspectos del Código País adopta y cuáles no (Superintendencia Financiera de Colombia, 2007). No obstante, a pesar de esta libertad de elección, algunas de estas disposiciones son de cumplimiento obligatorio, por ejemplo, la Resolución 275 de 2001 de la Superintendencia de Valores define algunas disposiciones obligatorias que deben cumplir los emisores de valores que esperan que sus títulos valores sean adquiridos por los fondos de pensiones.

En este sentido, Barbesta, Giudici, \& Lugo (2010) sugieren que con el objetivo de cumplir los requerimientos del mercado necesarios para realizar emisiones de valores las empresas italianas siguen comportamientos isomorfos antes de cotizar en la Bolsa de Valores. Por su parte, Holder-Webb \& Cohen (2012) proponen que el contenido vago y estilizado de los códigos de ética constituye una respuesta racional a la regulación que establece la existencia de dichos códigos. Los reguladores esperan que los códigos de ética sean diferentes de acuerdo a las cuestiones éticas, que son particulares de cada empresa. No obstante, Holder-Webb \& Cohen (2012) proporcionan evidencia de que la estructura, el contenido y el lenguaje de los códigos es consistente con una respuesta generalizada a presiones isomorfas. El estudio de De Lima, Oliveira, Ponte \& Rebouças (2016) encontró que los fondos de pensiones brasileños tienden a adoptar y difundir en mayor medida las prácticas de gobierno definidas en la ley, en tanto que, en las prácticas de adopción voluntaria encontraron altos niveles de dispersión, lo cual sugiere la existencia de isomorfismo coercitivo. De Lima et al. (2016) también encontraron evidencia que sugiere la presencia de isomorfismo mimético relacionado con el nivel de activos y la edad de los fondos de pensiones, los resultados mostraron que a mayor edad y mayor nivel de activos la dispersión es menor en la adopción de prácticas de gobierno corporativo voluntarias. 
A partir de estos estudios se formula la siguiente hipótesis:

$\mathrm{H}_{2}$ : Cuanto mayor sea el nivel de regulación en las prácticas de gobierno corporativo mayor será la homogeneización en la implementación de dichas prácticas en Colombia.

El cambio organizacional isomorfo de tipo mimético se presenta, según Powell \& Dimaggio (1991), cuando se entienden poco las tecnologías organizacionales o cuando el ambiente genera incertidumbre, entonces las organizaciones pueden construirse siguiendo el modelo de otras organizaciones similares en su campo, las cuales les parecen más legítimas o exitosas. En este sentido, (Dias Filho \& Moura, 2013) evalúan el grado en que las políticas de divulgación de información contable adoptadas por algunas instituciones financieras en Brasil están influenciadas por las prácticas de divulgación usadas por sus pares más grandes, encontrando que en ambientes de incertidumbre y ambigüedad las organizaciones se modelan imitando a otras que son percibidas como eficientes. En este mismo sentido, Zakaria (2012) sugiere que el proceso de fijación de la remuneración de los ejecutivos en empresas de Reino Unido sigue comportamientos de isomorfismo en ausencia de directrices claras y concretas en cuanto a lo que se considera aceptable.

El presente estudio desea validar la siguiente hipótesis:

$H_{3}$ : Las empresas consideradas exitosas (mayores niveles de ventas, mayores niveles de activos) se convierten en referentes para otras empresas al momento de adoptar prácticas de gobierno corporativo, por lo tanto, la implementación de dichas prácticas tiende hacia las prácticas por las que han optado las empresas exitosas.

\section{Metodología}

\subsection{Datos y muestra}

La información sobre implementación de prácticas de gobierno corporativo proviene de la encuesta Código País. Esta encuesta debe ser diligenciada por todas las empresas inscritas en el Registro Nacional de Valores y Emisores de Colombia (RNVE). La información de Ventas es obtenida de la Superintendencia Financiera de Colombia (SFC). La muestra comprende 735 registros-año correspondientes a un panel de datos balanceado de 105 empresas que respondieron la encuesta ${ }^{5}$ en todos los años del periodo 2008-2014. 64 empresas son empresas no familiares (ENF) y 41 son empresas familiares (EF).

\subsection{Modelo de investigación}

La medición de gobierno corporativo se hace mediante el índice de gobierno corporativo Código País (IGCCP) construido por Lagos \& Vecino (2014). Este índice evalúa el cumplimiento de 41 prácticas de gobierno corporativo que se encuentran definidas en el Código País ${ }^{6}$. Este código incluye 41 recomendaciones de gobierno corporativo que hacen referencia a cuatro grupos de prácticas: asamblea general de accionistas (11), junta directiva (15), revelación de información financiera y no financiera (13) y resolución de controversias (2). El IGCCP es un índice simple (no ponderado) que asigna el valor de uno (1) cuando la recomendación de gobierno corporativo es implementada por la empresa y cero (0) en caso contrario, de esta manera, el índice puede tomar valores en cero (0) y cuarenta y uno (41), donde un mayor valor indica mejores prácticas de gobierno corporativo. Para identificar si en la implementación de prácticas de gobierno corporativo de las empresas inscritas en el RNVE se presentan comportamientos isomorfos se estudia la dispersión en la implementación de prácticas de gobierno a través del coeficiente de variación y la varianza del IGCCP.

Siguiendo a Gómez-Betancourt, López, Betancourt \& Millán (2012) las empresas son clasificadas como EF cuando se identificó más del 50 por ciento de la

5 La muestra representa el $75 \%$ de las empresas que reportaron la encuesta de gobierno corporativo Código País en el 2014.

$6 \quad$ Las prácticas de gobierno corporativo incluidas en el Código País para el mercado colombiano pueden ser consultadas en: https://www.superfinanciera.gov.co/SFCant/Codigopais/ textos/codigopias.pdf 
propiedad en manos de hasta tres familias, en caso contrario son clasificadas como ENF. El isomorfismo coercitivo es estudiado clasificando las empresas de acuerdo a su situación en la Bolsa de Valores de Colombia. Las empresas se clasifican como Emisores, si durante el periodo 2008-2014 realizaron alguna emisión de bonos o acciones, en caso contrario son clasificadas como NO Emisores. Para estudiar la existencia de isomorfismo mimético se adopta una visión de éxito empresarial relacionada con el crecimiento. De acuerdo con Whetten (1987) el crecimiento es señal de una correcta administración a lo largo del tiempo, por lo tanto, un mayor tamaño es una característica deseable de cualquier organización. Bajo este enfoque y siguiendo los estudios de Dunne \& Hughes (1994) y Petrakis (1997) se usa el tamaño de la empresa (volumen de ventas) como una aproximación del éxito empresarial. Las empresas se agrupan de acuerdo a: si se ubican en el percentil 75 en Ventas durante 2008-2014, este grupo de empresas se consideran exitosas y son denominamos Top Ventas. Las empresas restantes se clasifican en EF NO Top Ventas y ENF NO Top Ventas.

\section{Resultados y discusión}

\section{1. ¿Existe homogeneización en las prácticas de gobierno corporativo en Colombia?}

Desde la entrada en vigencia del Código País en 2007, muchas empresas en Colombia se han comprometido con la implementación de buenas prácticas de gobierno. Esta situación se evidencia en el Gráfico 1, donde se muestra que durante el periodo 2008-2014 las empresas han mejorado en las mediciones promedio, tanto EF como ENF presentan mejores mediciones cada año.

Gráfico 1. Evolución promedio del IGCCP para el periodo 2008-2014

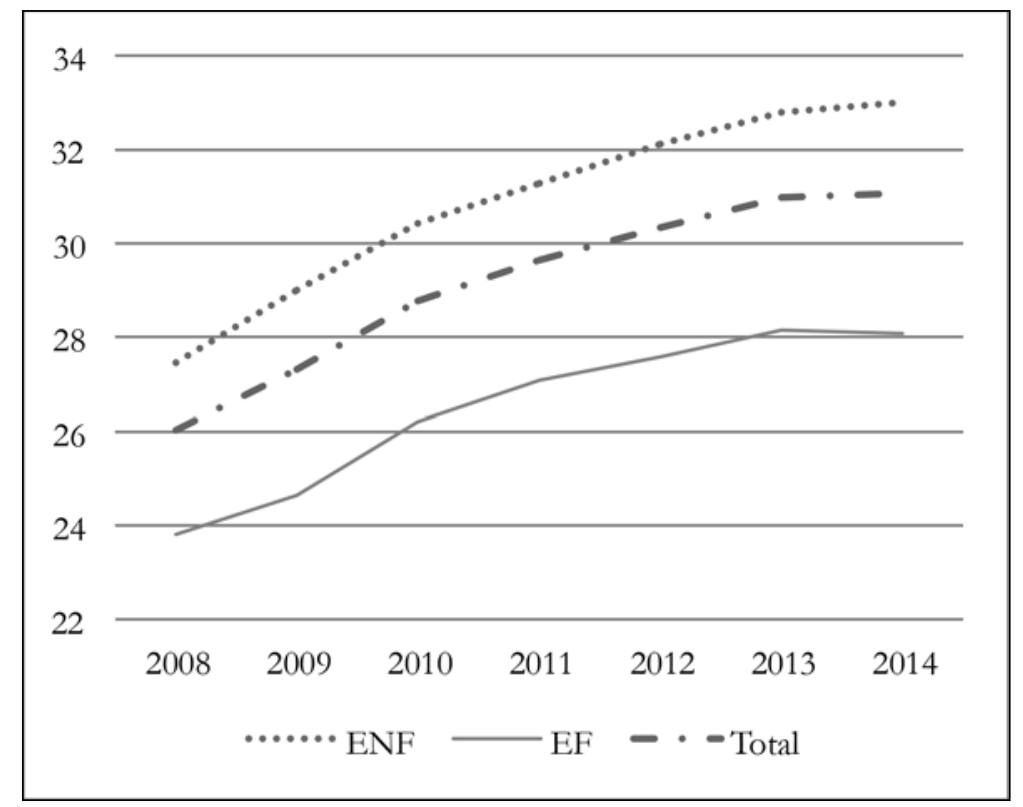

Fuente: Elaboración de los autores basado en este estudio. 
En la Tabla 1 se presentan los principales estadísticos descriptivos (mínimo, máximo, media, varianza, coeficiente de variación) para el comportamiento del IGCCP. Se resalta que el proceso de implementación de prácticas de gobierno corporativo en Colombia va por buen camino, los avances en la medición son significativos si se comparan entre 2008 y 2014. En la fila 2008-2014 de la Tabla 1, se hace una comparación de la media del IGCCP al inicio y al final del periodo de estudio, tanto para las EF y ENF como para la muestra total. Se evidencia que tanto ENF como EF han mejorado sus estándares de gobierno corporativo y que estas mejoras son significativas. Específicamente, las ENF han mejorado en promedio 5.5 unidades de gobierno corporativo entre 2008 y
2014, las EF han aumentado la medición de gobierno en promedio 4.26 unidades, en tanto que en la muestra total el cambio fue de 5.04 unidades.

Se evidencia que las ENF tienen mejores estándares de gobierno corporativo, la prueba t muestra que la diferencia de medias entre ENF y EF es significativa para todos los años del estudio, esta diferencia tiende a ampliarse cada año. En el 2008 la diferencia promedio de la medición era de 3.64, para 2014 esta diferencia crece a 4.96. Los resultados son diferentes de acuerdo al carácter familiar, esto puede indicar que en las EF la adopción de nuevas prácticas de gobierno corporativo es un proceso que se hace a menor ritmo.

Tabla 1. Gobierno corporativo en EF y ENF en Colombia 2008-2014

\begin{tabular}{|c|c|c|c|c|c|c|c|c|c|c|c|c|c|c|c|c|}
\hline & \multicolumn{5}{|c|}{ ENF (64) } & \multicolumn{5}{|c|}{ EF (41) } & \multicolumn{5}{|c|}{ Total (105) } & \multirow{2}{*}{$\begin{array}{c}\text { Comparación } \\
\text { de Medias }\end{array}$} \\
\hline & Min. & Max. & Media & Var & CV & Min. & Max. & Media & Var & CV & Min. & Max. & Media & Var & CV & \\
\hline \multirow[t]{2}{*}{2008} & 11.50 & 40.50 & 27.45 & 56.20 & 0.273 & 12.23 & 39.67 & 23.81 & 34.01 & 0.245 & 11.50 & 40.50 & 26.03 & 50.31 & 0.272 & $3.64^{\star \star \star}$ \\
\hline & & & & & & & & & & & & & & & & $(2.78)$ \\
\hline \multirow[t]{2}{*}{2009} & 10.50 & 40.50 & 29.00 & 49.19 & 0.242 & 11.33 & 40.17 & 24.65 & 35.79 & 0.243 & 10.50 & 40.50 & 27.31 & 48.11 & 0.254 & $4.35^{\star \star \star}$ \\
\hline & & & & & & & & & & & & & & & & $(3.39)$ \\
\hline \multirow[t]{2}{*}{2010} & 10.50 & 41.00 & 30.42 & 46.98 & 0.225 & 16.33 & 39.50 & 26.20 & 33.84 & 0.222 & 10.50 & 41.00 & 28.78 & 45.76 & 0.235 & $4.22^{\star \star \star}$ \\
\hline & & & & & & & & & & & & & & & & $(3.38)$ \\
\hline \multirow[t]{2}{*}{2011} & 11.33 & 40.50 & 31.27 & 45.05 & 0.215 & 16.08 & 40.50 & 27.09 & 34.98 & 0.218 & 11.33 & 40.50 & 29.64 & 44.95 & 0.226 & $4.18^{\star \star \star}$ \\
\hline & & & & & & & & & & & & & & & & $(3.35)$ \\
\hline \multirow[t]{2}{*}{2012} & 9.33 & 40.50 & 32.11 & 43.61 & 0.206 & 15.75 & 40.50 & 27.58 & 36.66 & 0.220 & 9.33 & 40.50 & 30.34 & 45.47 & 0.222 & $4.54^{\star \star \star}$ \\
\hline & & & & & & & & & & & & & & & & $(3.62)$ \\
\hline \multirow[t]{2}{*}{2013} & 14.83 & 41.00 & 32.79 & 42.32 & 0.198 & 17.75 & 41.00 & 28.15 & 37.71 & 0.218 & 14.83 & 41.00 & 30.98 & 45.31 & 0.217 & $4.64^{\star \star \star}$ \\
\hline & & & & & & & & & & & & & & & & $(3.69)$ \\
\hline \multirow[t]{2}{*}{2014} & 15.33 & 41.00 & 33.00 & 40.05 & 0.192 & 16.08 & 41.00 & 28.07 & 40.50 & 0.227 & 15.33 & 41.00 & 31.08 & 45.68 & 0.217 & $4.93^{\star \star *}$ \\
\hline & & & & & & & & & & & & & & & & $(3.88)$ \\
\hline \multirow[t]{2}{*}{$\begin{array}{l}2008- \\
2014\end{array}$} & 3.83 & 0.50 & $5.55^{\star * *}$ & -16.14 & -0.08 & 3.85 & 1.33 & $4.26^{\star \star \star}$ & 6.49 & -0.02 & 3.83 & 0.50 & $5.04^{\star \star \star}$ & -4.63 & -0.06 & \\
\hline & & & $(8.88)$ & $(1.42)$ & & & & $(7.75)$ & $(0.84)$ & & & & (11.49) & $(1.11)$ & & \\
\hline
\end{tabular}

Notas: El IGCCP puede tomar valores entre cero (0) y cuarenta y uno (41), donde un mayor valor indica mejores prácticas de gobierno corporativo, esta tabla muestra los principales estadísticos descriptivos para el IGCCP: Mínimo (Min), Máximo (Max), Media, Varianza (Var) y Coeficiente de Variación (CV). El CV está expresado en notación decimal. El análisis se realiza en un panel de datos balanceado de 105 empresas, de las cuales 64 son ENF y 41 son EF. En la prueba t de diferencias de medias se muestra el valor de la diferencia y entre paréntesis el valor del estadístico de prueba. ${ }^{* * * *}$ indica significancia estadística a nivel del $1 \%$.

Fuente: Elaboración de los autores basado en este estudio. 
Para identificar si en el proceso de implementación de prácticas de gobierno corporativo se presentan comportamientos isomorfos, analizamos el coeficiente de variación y la varianza (anteriormente pre- sentado en la Tabla 1). En el gráfico 2 se observa que la dispersión se tiende a reducir con el tiempo. No obstante, se evidencia que las ENF y EF presentan comportamientos diferentes.

Gráfico 2. Tendencia en el coeficiente de variación del IGCCP para el periodo 2008-2014

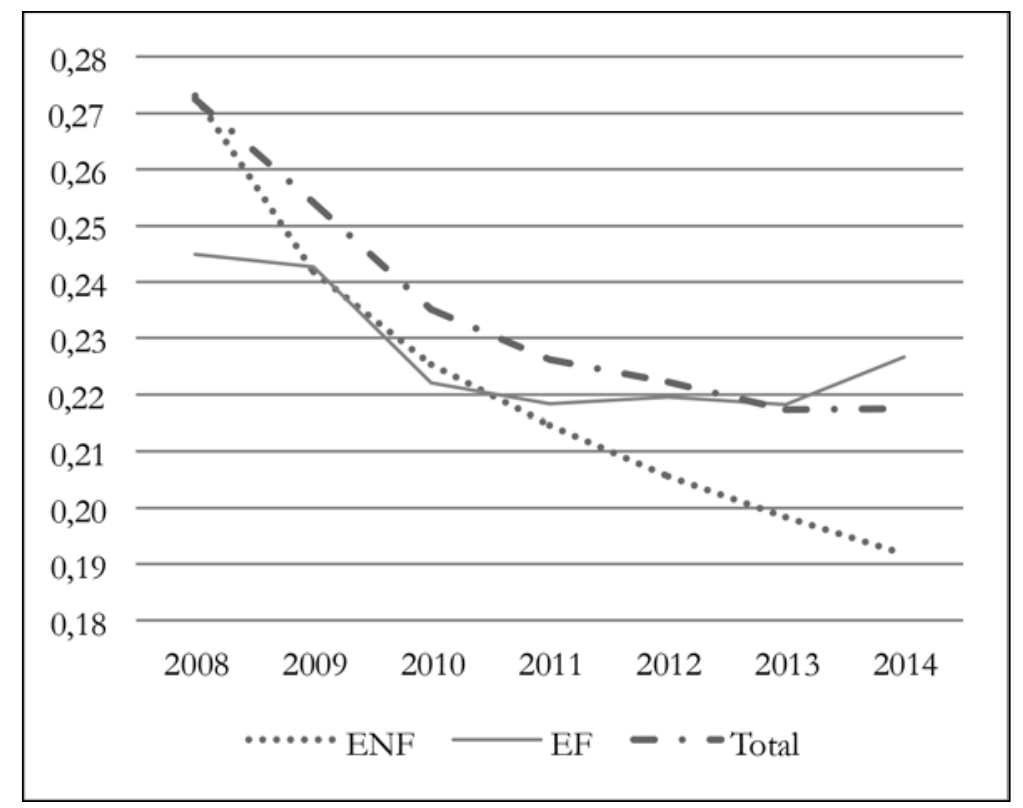

Fuente: Elaboración de los autores basado en este estudio.

Para el total de la muestra, el coeficiente de variación se reduce en 5.5 puntos porcentuales, pasa de 0.272 en el 2008 a 0.217 en el 2014. Esto sugiere que puede existir una tendencia hacia la homogeneización en la implementación de prácticas de gobierno corporativo. El análisis de acuerdo al carácter familiar, muestra que la tendencia hacia la homogeneización está impulsada por el grupo de ENF donde el coeficiente de variación se reduce de 0.273 a 0.192 . Un comportamiento diferente se tiene en las $\mathrm{EF}$, el coeficiente de variación presenta una tendencia a la baja hasta el 2011 donde alcanza una medición de 0.218. Después de 2011 el coeficiente de variación oscila hacia arriba y hacia abajo, hasta ubicarse en 0.227 en 2014 , valor que significa una reducción de 1.9 puntos porcentuales comparado con el valor registrado en 2008 . Es importante destacar que al inicio del periodo de estudio las EF tenían un coeficiente de variación menor que el encontrado en las ENF, es decir, las EF eran el grupo más homogéneo en 2008. Sin embargo, esta situación se revierte al final del estudio, donde las ENF son más homogéneas en la medición de gobierno corporativo.

Para identificar si la tendencia decreciente, observada en el coeficiente de variación, indica la existencia de isomorfismo en la implementación de prácticas de gobierno corporativo se aplica una prueba que compara la varianza inicial de cada grupo con la encontrada al final del periodo. En la muestra total la varianza se reduce de 50.31 a 45.68, una 
reducción de 4.63 unidades. Sin embargo, la prueba de varianzas muestra que esta diferencia no es significativa. Respecto a las EF se tiene que la varianza aumenta en 6.49 unidades entre 2008 y 2014. Finalmente, aunque en las ENF se encuentra una reducción mayor en la varianza (16.14 unidades) la prueba muestra que dicha diferencia no es significativa.

Los resultados anteriores indican que se debe rechazar $H_{1 a}$. Aunque se evidencia que la dispersión tiende a reducirse en las ENF, dicha reducción no es significativa. Estos resultados difieren con lo planteado por Powell \& Dimaggio (1991), quienes afirman que en etapas iniciales, los campos organizacionales presentan una diversidad de enfoques y formas, sin embargo, a medida que el campo organizacional se va consolidando surgen fuerzas que las hacen cada vez más similares, tendiendo hacia la homogeneización en muchas de sus formas y prácticas. Es posible que en un estudio con horizonte de tiempo más amplio se puedan identificar comportamientos isomorfos en la implementación de prácticas de gobierno corporativo, consideramos que un rango de siete años es limitado para observar procesos de homogeneización.

Respecto a $H_{1 b}$ se puede afirmar que es cierta, los resultados muestran que el comportamiento en la dispersión es diferente en las EF. En las ENF la dispersión es estable (se reduce periodo a periodo). En tanto que en las EF la dispersión se comporta diferente, es inestable (algunos años aumenta y en otros se reduce).

\section{2. ¿Influye la regulación en la implementa- ción de prácticas de gobierno corporativo en Colombia?}

El Código País es una compilación de prácticas de gobierno corporativo dirigidas a las empresas que son o que aspiran a ser emisores de valores en el mercado colombiano. Teniendo en cuenta esto, adicional a la clasificación de EF y ENF, clasificamos a las empresas como Emisores si durante el periodo 2008-2014 realizaron alguna emisión de bonos o acciones. En caso contrario son clasificadas como NO Emisores.

Las empresas que han emitido valores en el mercado colombiano se someten a una mayor regulación en cuanto a gobierno corporativo7. El hecho de que existan algunas prácticas obligatorias para los Emisores sugiere que este grupo debería tender hacia mayores estándares de gobierno corporativo (isomorfismo coercitivo). Mediante una prueba t se compara la media del IGCCP de los Emisores y los NO Emisores, los resultados muestran que en promedio los Emisores tienen mayores mediciones de gobierno corporativo. Las diferencias en la media del IGCCP son significativas en todos los años del estudio. Esto sugiere que ser Emisor podría modificar los resultados mostrados en el numeral 4.1 donde se encontró que las EF presentan menores estándares de gobierno corporativo. En la tabla 2 se presentan un ANOVA que compara la media del IGCCP entre cuatro grupos de empresas (ENF Emisor, EF Emisor, ENF NO Emisor, EF NO Emisor).

7 La Resolución 275 de 2001 establece una serie de requisitos que deben cumplir los emisores de valores como condición para que los fondos de pensiones puedan invertir en sus títulos. Entre otros se tiene: i) los emisores deberán tener colocadas entre inversionistas diferentes del grupo o personas que los controle al menos el $20 \%$ del total de sus acciones; ii) mecanismos para garantizar los derechos de todos los accionistas e inversores; $y$ iii) parámetros mínimos de divulgación de información que debe ser entregada a accionistas, al mercado y al público en general. 
Tabla 2. IGCCP promedio de acuerdo al carácter de Emisor o NO Emisor entre 2007-2014

\begin{tabular}{|c|c|c|c|c|c|c|c|c|c|c|}
\hline & & \multirow{3}{*}{$\begin{array}{l}\text { EF NO } \\
\text { Emisor } \\
(27)\end{array}$} & \multirow{3}{*}{$\begin{array}{l}\text { ENF } \\
\text { Emisor } \\
(32)\end{array}$} & \multirow{3}{*}{$\begin{array}{c}\text { EF } \\
\text { Emisor } \\
(14)\end{array}$} & \multirow{3}{*}{$\begin{array}{l}\text { NO } \\
\text { Emisor } \\
(59)\end{array}$} & \multirow{3}{*}{$\begin{array}{c}\text { Emisor } \\
(46)\end{array}$} & \multirow{2}{*}{\multicolumn{3}{|c|}{$\begin{array}{c}\text { ANOVA } \\
\text { ENF Emisor }\end{array}$}} & \multirow{3}{*}{$\begin{array}{c}\text { t-student } \\
\text { Emisor } \\
\text { NO } \\
\text { Emisor }\end{array}$} \\
\hline & \multirow{2}{*}{$\begin{array}{c}\text { ENF NO } \\
\text { Emisor } \\
\text { (32) }\end{array}$} & & & & & & & & & \\
\hline & & & & & & & $\begin{array}{c}\text { EF } \\
\text { Emisor }\end{array}$ & $\begin{array}{l}\text { EF NO } \\
\text { Emisor }\end{array}$ & $\begin{array}{l}\text { ENF NO } \\
\text { Emisor }\end{array}$ & \\
\hline \multirow[t]{2}{*}{2008} & 23.84 & 23.06 & 31.07 & 25.27 & 23.48 & 29.31 & $5.81^{\star \star}$ & $8.02^{\star \star \star}$ & $7.24^{\star * *}$ & $5.83^{\star \star \star}$ \\
\hline & & & & & & & & & & $(4.56)$ \\
\hline \multirow[t]{2}{*}{2009} & 26.02 & 23.88 & 31.99 & 26.15 & 25.04 & 30.21 & $5.85^{\star *}$ & $8.11^{\star \star \star}$ & $5.97^{\star * *}$ & $5.17^{\star \star \star *}$ \\
\hline & & & & & & & & & & $(4.06)$ \\
\hline \multirow[t]{2}{*}{2010} & 27.28 & 25.55 & 33.57 & 27.45 & 26.49 & 31.71 & $6.11^{* \star}$ & $8.01^{\star \star \star}$ & $6.28^{\star * \star}$ & $5.22^{\star \star \star}$ \\
\hline & & & & & & & & & & $(4.22)$ \\
\hline \multirow[t]{2}{*}{2011} & 27.79 & 26.28 & 34.76 & 28.65 & 27.10 & 32.90 & $6.11^{\star \star \star}$ & $8.48^{\star \star \star}$ & $6.98^{\star * \star}$ & $5.80^{\star \star \star \star}$ \\
\hline & & & & & & & & & & $(4.86)$ \\
\hline \multirow[t]{2}{*}{2012} & 28.39 & 26.71 & 35.83 & 29.24 & 27.63 & 33.83 & 6.59 *** & $9.12^{\star \star \star}$ & $7.44^{* * *}$ & $6.20^{* * *}$ \\
\hline & & & & & & & & & & (5.24) \\
\hline \multirow[t]{2}{*}{2013} & 29.05 & 27.34 & 36.53 & 29.72 & 28.27 & 34.46 & $6.81^{* \star *}$ & $9.19^{\star \star \star}$ & $7.48^{\star \star \star}$ & $6.19^{\star \star \star}$ \\
\hline & & & & & & & & & & (5.24) \\
\hline \multirow[t]{2}{*}{2014} & 29.55 & 27.38 & 36.45 & 29.40 & 28.56 & 34.31 & $7.06^{\star \star \star}$ & $9.07^{\star \star \star}$ & $6.91 * * \star$ & $5.75^{\star \star \star}$ \\
\hline & & & & & & & & & & $(4.75)$ \\
\hline
\end{tabular}

Notas: Esta tabla muestra el comportamiento promedio del IGCCP para EF y ENF teniendo en cuenta si las empresas han o NO emitido bonos y/o acciones durante el periodo 2007-2014. Mediante un ANOVA se compara a media entre cuatro grupos de empresas (ENF Emisor, EF Emisor, ENF NO Emisor, EF NO Emisor). En los resultados del ANOVA solo se presentan las diferencias de medias entre los grupos que son significativas. Mediante una prueba t-student se compara la media entre Emisores y NO Emisores, sin incluir el carácter familiar. La prueba t presenta el valor de la diferencia entre las medias y entre paréntesis el valor del estadístico de prueba. ${ }^{* * * *},{ }^{* *}$, indican significancia estadística a nivel de $1 \%$ y $5 \%$, respectivamente.

Fuente: Elaboración de los autores basado en este estudio.

Los resultados muestran que no existen diferencias significativas entre EF Emisor, ENF NO Emisor y EF NO Emisor (en cualquiera de sus combinaciones). En tanto que las ENF Emisores se diferencian de estos tres grupos anteriores, las ENF Emisores presentan los mayores estándares de gobierno corporativo siendo significativas las diferencias en todos los años del estudio. Se destaca que las mayores diferencias se presentan con las EF NO Emisores, con este grupo la diferencia era de 8.02 unidades en 2008 y para 2014 crece a 9.07. En comparación con las EF Emisores, la diferencia también aumenta al final del estudio, pasa de 5.81 en 2008 a 7.06 en 2014. Frente a las ENF NO Emisores la diferencia presenta comportamientos inestables, en 2008 era de 7.24 y finaliza con 6.91 en 2014.
En la tabla 3 se muestra el comportamiento de la dispersión teniendo en cuenta los cuatro grupos de empresas considerados anteriormente. El coeficiente de variación muestra una tendencia decreciente tanto en Emisores como en NO Emisores, no obstante, es mayor la reducción en los Emisores donde la dispersión disminuye en 5.8 puntos porcentuales en comparación con 4.9 puntos porcentuales de disminución en los NO Emisores. Esto puede indicar que entre mayor regulación mayor será la tendencia hacia la homogenización. Sin embargo, cuando se incorpora al análisis la clasificación de ENF y EF, se observa que la reducción en la variación es similar entre las ENF, independiente de si son Emisores o NO Emisores. 
Tabla 3. Comportamiento de la dispersión del IGCCP de acuerdo al carácter de Emisor o NO Emisor entre 2007-2014

\begin{tabular}{|c|c|c|c|c|c|c|c|c|c|c|c|c|c|c|c|c|c|c|}
\hline & \multicolumn{3}{|c|}{$\begin{array}{l}\text { ENF NO Emisor } \\
\left(\begin{array}{l}(32)\end{array}\right.\end{array}$} & \multicolumn{3}{|c|}{$\begin{array}{l}\text { EF NO Emisor } \\
(27)\end{array}$} & \multicolumn{3}{|c|}{ ENF Emisor (32) } & \multicolumn{3}{|c|}{ EF Emisor (14) } & \multicolumn{3}{|c|}{ NO Emisor (59) } & \multicolumn{3}{|c|}{ Emisor (46) } \\
\hline & Media & Var & CV & Media & Var & CV & Media & Var & CV & Media & Var & CV & Media & Var & CV & Media & Var & CV \\
\hline 2008 & 23.84 & 46.85 & 0.287 & 23.06 & 38.60 & 0.269 & 31.07 & 40.33 & 0.204 & 25.27 & 23.98 & 0.194 & 23.48 & 42.50 & 0.278 & 29.31 & 42.01 & 0.221 \\
\hline 2009 & 26.02 & 49.03 & 0.269 & 23.88 & 42.94 & 0.274 & 31.99 & 32.52 & 0.178 & 26.15 & 20.59 & 0.174 & 25.04 & 46.61 & 0.273 & 30.21 & 35.75 & 0.198 \\
\hline 2010 & 27.28 & 43.85 & 0.243 & 25.55 & 38.44 & 0.243 & 33.57 & 31.23 & 0.166 & 27.45 & 24.69 & 0.181 & 26.49 & 41.42 & 0.243 & 31.71 & 36.73 & 0.191 \\
\hline 2011 & 27.79 & 42.25 & 0.234 & 26.28 & 40.01 & 0.241 & 34.76 & 24.19 & 0.141 & 28.65 & 23.64 & 0.170 & 27.10 & 41.09 & 0.237 & 32.90 & 31.58 & 0.171 \\
\hline 2012 & 28.39 & 41.99 & 0.228 & 26.71 & 42.86 & 0.245 & 35.83 & 18.07 & 0.119 & 29.24 & 22.57 & 0.162 & 27.63 & 42.37 & 0.236 & 33.83 & 28.39 & 0.158 \\
\hline 2013 & 29.05 & 37.82 & 0.212 & 27.34 & 42.82 & 0.239 & 36.53 & 19.31 & 0.120 & 29.72 & 26.36 & 0.173 & 28.27 & 40.15 & 0.224 & 34.46 & 30.95 & 0.161 \\
\hline 2014 & 29.55 & 37.56 & 0.207 & 27.38 & 47.94 & 0.253 & 36.45 & 19.22 & 0.120 & 29.40 & 25.86 & 0.173 & 28.56 & 42.75 & 0.229 & 34.31 & 31.49 & 0.164 \\
\hline \multirow[t]{2}{*}{$2008-$} & $5.72^{\star \star \star}$ & -9.280 & -0.080 & $4.33^{\star \star *}$ & 9.34 & -0.017 & $5.38^{* \star *}$ & $-21.11^{\star \star}$ & -0.084 & $4.13^{\star \star \star}$ & 1.88 & -0.021 & $5.08^{\star \star \star *}$ & 0.25 & -0.049 & $5.00 \star \star \star$ & -10.51 & -0.058 \\
\hline & $(6.33)$ & (1.25) & & $(6.97)$ & $(0.81)$ & & $(6.15)$ & $(2.15)$ & & $(3.71)$ & $(0.93)$ & & $(8.93)$ & $(0.99)$ & & $(7.20)$ & (1.35) & \\
\hline
\end{tabular}

Notas: Esta tabla muestra algunos estadísticos descriptivos (Media, Varianza (Var) y Coeficiente de Variación (CV)) para el IGCCP combinando los criterios de Emisor o NO Emisor y de Familiar o NO Familiar. El CV está expresado en notación decimal. Tanto en la prueba de diferencia de medias como en la prueba de diferencia de varianzas, se presenta el valor de la diferencia y entre paréntesis el valor del estadístico de prueba. ${ }^{* * * *},{ }^{* * *}$, indican significancia estadística a nivel de $1 \%$ y $5 \%$, respectivamente.

Fuente: Elaboración de los autores basado en este estudio.

El coeficiente de variación en ENF NO Emisores se reduce de 0.287 en 2008 a 0.207 en 2014 (8 puntos porcentuales), en tanto que en los ENF Emisores la reducción es de 8.4 puntos porcentuales, en este último grupo el coeficiente de variación pasa de 0.204 a 0.120. Respecto a las EF se encuentra que en las EF NO Emisores la dispersión es mayor que en las EF Emisores, no obstante, la reducción en la dispersión es similar en los dos grupos. En las
EF NO Emisores la dispersión pasa de 0.269 en 2008 a 0.253 en 2014 (reducción de 1.7 puntos porcentuales). En tanto que en las EF Emisores la dispersión se mantiene en niveles estables, luego de pequeños cambios en el periodo de estudio, en 2008 la dispersión era de 0.194 y termina en 2014 con 0.173 (reducción de 2.1 puntos porcentuales). En el gráfico 3 se resume el comportamiento del coeficiente de variación. 
Gráfico 3. Tendencia en el coeficiente de variación del IGCCP para el periodo 2008-2014 combinando las clasificaciones de Emisor y Familiar

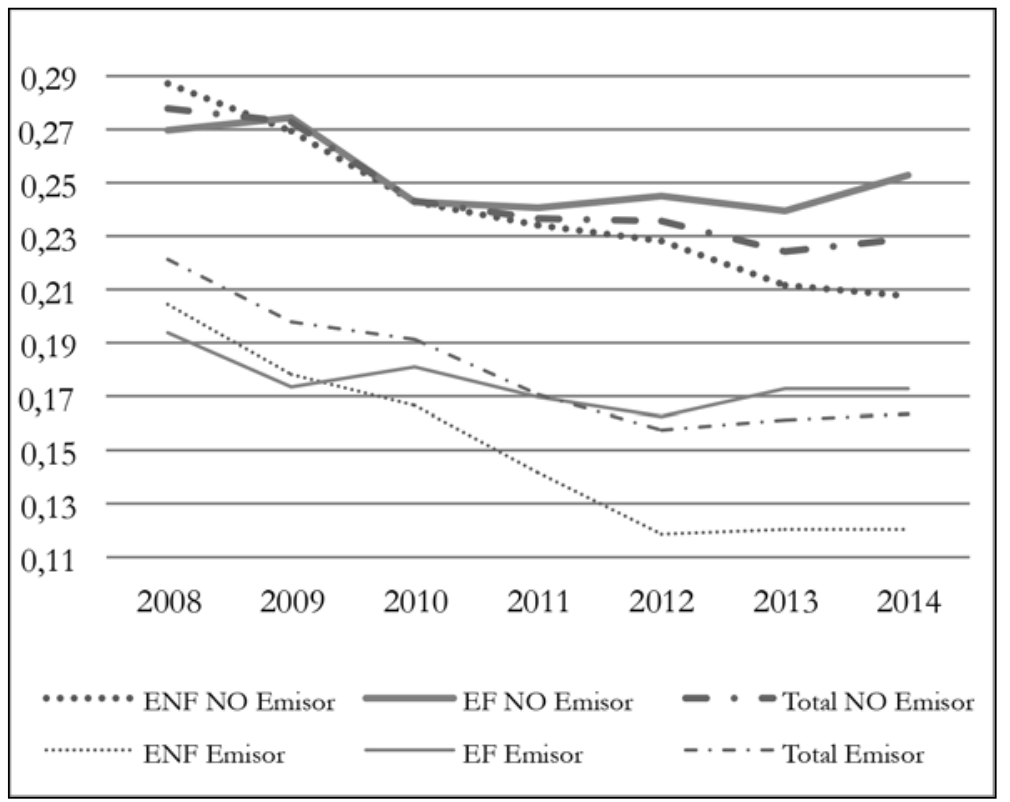

Fuente: Elaboración de los autores basado en este estudio.

En la fila 2008-2014 de la Tabla 3, se hace una comparación de la media del IGCCP al inicio y al final del periodo de estudio para las empresas agrupadas como Emisores o NO Emisores y para los cuatro grupos derivados de añadir el carácter familiar al carácter de Emisor (ENF Emisor, EF Emisor, ENF NO Emisor, EF NO Emisor). Los resultados confirman lo encontrado en el numeral 4.1 donde se muestra que los estándares de gobierno corporativo vienen mejorando con el tiempo. Las diferencias entre las mediciones de 2008 y las de 2014 son significativas en todos los grupos. El mayor aumento se presenta en las ENF NO Emisores (5.72 unidades), seguido de los ENF Emisores (5.38), en las EF NO Emisores la diferencia es de 4.33 unidades, y finalmente, el grupo con menor aumento en el IGCCP son las EF Emisores con 4.13. Al clasificar las empresas solo por el carácter emisor, se encuentra que el aumento es similar tanto en Emisores como en NO Emisores, 5 unidades y 5.08 unidades respectivamente.
Respecto a la varianza, en la fila 2008-2014 de la tabla 3 se compara la varianza en 2008 con la encontrada al final del estudio. Se encuentra que en los Emisores se reduce en 10.514 unidades y en los NO Emisores aumenta 0.253 unidades, sin embargo, ninguno de estos cambios es significativo. Comparando con los dos criterios (Emisor, Familiar) se tiene que las ENF Emisores son el grupo que más reduce la dispersión, la varianza se reduce en 21.11 unidades siendo el único grupo donde la reducción en la dispersión es significativa (a nivel del 5\%). En los EF NO Emisores la dispersión aumenta en 9.34, en las EF Emisores aumenta 1.88 y en las ENF NO Emisores se reduce 9.28 unidades.

Estos resultados sugieren que en las ENF Emisores existe una tendencia hacia la homogeneización en la implementación de prácticas de gobierno corporativo, no obstante, los demás análisis conllevan 
a rechazar $\mathrm{H}_{2}$ ya que la regulación no es un factor significativo en la reducción de la dispersión. Los resultados también permiten identificar que la dispersión en la implementación de prácticas de gobierno corporativo es diferente en EF y ENF, esto es consistente con lo encontrado en el numeral 4.1 donde se observó una diferencia en la implementación de prácticas de gobierno corporativo entre EF y ENF. Creemos que la regulación en gobierno corporativo no es un factor determinante en la reducción de la dispersión debido a que, aunque existen prácticas de gobierno corporativo obligatorias, éstas pueden no estar bien definidas. Abbott \& Snidal (2000) plantean tres dimensiones para distinguir entre una norma de tipo Soft (Soft Law) y una de tipo Hard (Hard Law): i) precisión de la norma; ii) obligatoriedad; y iii) delegación en un tercero de la toma de decisiones. La definición de Abbott \& Snidal indica que Hard Law es una disposición vinculante, precisa y que ha sido delegada a un tercero para su interpretación y verificación de su cumplimiento. Consideramos que la mayor parte de las normas de gobierno corporativo en Colombia son normas que son débiles a lo largo de al menos una de estas tres dimensiones. Esto hace que las presiones sean menores en las empresas que deben adoptar dichas normas. Por otro lado, es probable que las empresas no presenten diferencias debido a que cumplen con la normatividad en los documentos oficiales, pero su comportamiento real no tiene relación con ello.
4.3. ¿Las empresas adoptan prácticas de gobierno corporativo teniendo como referentes a otras empresas que consideran exitosas en el medio?

Para estudiar la existencia de isomorfismo mimético en la implementación de prácticas de gobierno corporativo se usa el tamaño de la empresa (volumen de ventas) como una aproximación del éxito empresarial. Las empresas se agrupan de acuerdo a si se ubican en el percentil 75 en Ventas durante 2008-2014, a este grupo de empresas las consideramos exitosas y las denominamos Top Ventas. Las empresas restantes se clasifican en ENF NO Top Ventas y EF NO Top Ventas. Para identificar si existen diferencias significativas entre los tres grupos definidos se aplica un ANOVA.

En la Tabla 4 se presentan los resultados. Estos muestran que las Top Ventas tienen mayores estándares de gobierno corporativo en comparación con las demás empresas. Sin embargo, las diferencias sólo son significativas cuando se comparan con las EF NO Top Ventas. En 2008 la diferencia entre las Top Ventas y las EF NO Top Ventas era de 7.14 unidades, en 2014 la diferencia crece a 8.82. Esto indica que las EF NO Top Ventas, a pesar de que mejoran sus mediciones de gobierno corporativo, lo hacen a un ritmo menor que en las Top Ventas. La misma situación se encuentra entre EF NO Top Ventas y ENF NO Top Ventas, estas últimas mejoran a un ritmo mayor.

Tabla 4. Comportamiento de la dispersión del IGCCP de acuerdo al volumen de ventas entre 2007-2014

\begin{tabular}{|c|c|c|c|c|c|c|c|c|c|c|c|}
\hline & & & & & & & & & & \multirow{2}{*}{\multicolumn{2}{|c|}{$\begin{array}{c}\text { Comparación de } \\
\text { Medias ANOVA }\end{array}$}} \\
\hline & \multicolumn{3}{|c|}{ Top Ventas (10) } & \multicolumn{3}{|c|}{ EF NO Top Ventas (37) } & \multicolumn{3}{|c|}{ ENF NO Top Ventas (58) } & & \\
\hline & Media & Var & cV & Media & Var & CV & Media & Var & CV & $\begin{array}{c}\text { Top } \\
\text { Ventas }\end{array}$ & $\begin{array}{l}\text { ENF NO Top } \\
\text { Ventas }\end{array}$ \\
\hline 2008 & 30.55 & 44.78 & 0.219 & 23.41 & 31.94 & 0.241 & 26.93 & 55.70 & 0.277 & $-7.14^{\star \star \star}$ & $-3.52^{\star \star}$ \\
\hline 2009 & 31.06 & 39.35 & 0.202 & 24.19 & 34.85 & 0.244 & 28.64 & 48.97 & 0.244 & $-6.87^{\star \star \star}$ & $-4.46^{\star \star \star}$ \\
\hline 2010 & 32.89 & 31.44 & 0.171 & 25.64 & 32.05 & 0.221 & 30.07 & 47.25 & 0.229 & $-7.25^{\star \star \star}$ & $-4.43^{* * *}$ \\
\hline 2011 & 33.61 & 30.90 & 0.165 & 26.55 & 32.91 & 0.216 & 30.93 & 45.73 & 0.219 & $-7.06^{\star \star \star}$ & $-4.38^{\star \star \star}$ \\
\hline 2012 & 35.02 & 21.75 & 0.133 & 26.99 & 35.48 & 0.221 & 31.68 & 44.17 & 0.210 & $-8.04^{\star \star \star}$ & $-4.69 * * *$ \\
\hline
\end{tabular}




\begin{tabular}{|c|c|c|c|c|c|c|c|c|c|c|c|}
\hline & & & & & & & & & & \multirow{2}{*}{\multicolumn{2}{|c|}{$\begin{array}{c}\text { Comparación de } \\
\text { Medias ANOVA }\end{array}$}} \\
\hline & \multicolumn{3}{|c|}{ Top Ventas (10) } & \multicolumn{3}{|c|}{ EF NO Top Ventas (37) } & \multicolumn{3}{|c|}{ ENF NO Top Ventas (58) } & & \\
\hline & Media & Var & CV & Media & Var & CV & Media & Var & CV & $\begin{array}{c}\text { Top } \\
\text { Ventas }\end{array}$ & $\begin{array}{c}\text { ENF NO Top } \\
\text { Ventas }\end{array}$ \\
\hline 2013 & 35.72 & 25.02 & 0.140 & 27.57 & 36.04 & 0.218 & 32.33 & 42.61 & 0.202 & $-8.15^{\star \star \star}$ & $-4.76^{\star \star \star}$ \\
\hline 2014 & 36.09 & 25.57 & 0.140 & 27.48 & 38.89 & 0.227 & 32.51 & 39.85 & 0.194 & $-8.62^{\star \star \star}$ & $-5.03^{\star \star \star}$ \\
\hline \multirow[t]{2}{*}{$\begin{array}{l}2008- \\
2014\end{array}$} & $5.55^{\star \star \star}$ & -19.21 & -0.079 & $4.07^{\star \star \star}$ & 6.96 & -0.015 & $5.58^{\star \star \star}$ & -15.85 & -0.083 & & \\
\hline & $(3.98)$ & $(1.75)$ & & $(7.08)$ & $(0.82)$ & & $(8.45)$ & $(1.41)$ & & & \\
\hline
\end{tabular}

Notas: Esta tabla muestra algunos estadísticos descriptivos (Media, Varianza (Var) y Coeficiente de Variación (CV)) para el IGCCP en Top Ventas, NO Top Ventas EF y NO Top Ventas ENF. El CV está expresado en notación decimal. En la prueba ANOVA se presenta solo el valor de la diferencia de medias entre los grupos. En la prueba t de diferencia de medias y de varianzas entre el año 2008 y 2014 se muestra el valor de la diferencia y entre paréntesis se muestra el valor del estadístico de prueba. ${ }^{* * * *},{ }^{* * *}$, indican significancia estadística a nivel de $1 \%$ y $5 \%$, respectivamente.

Fuente: Elaboración de los autores basado en este estudio

Respecto a la variabilidad, el coeficiente de variación presenta una tendencia decreciente en los tres grupos (Top Ventas, ENF NO Top Ventas y EF NO Top Ventas). No obstante, el comportamiento es menos marcado el las EF NO Top Ventas. Estos resultados son consistentes con los encontrados en los numerales 4.1 y 4.2 donde se muestra que las empresas familiares presentan una dinámica diferente en la implementación de prácticas de gobierno corporativo.

Gráfica 4. Tendencia en el coeficiente de variación del IGCCP para el periodo 2008-2014 de acuerdo con los niveles de ventas

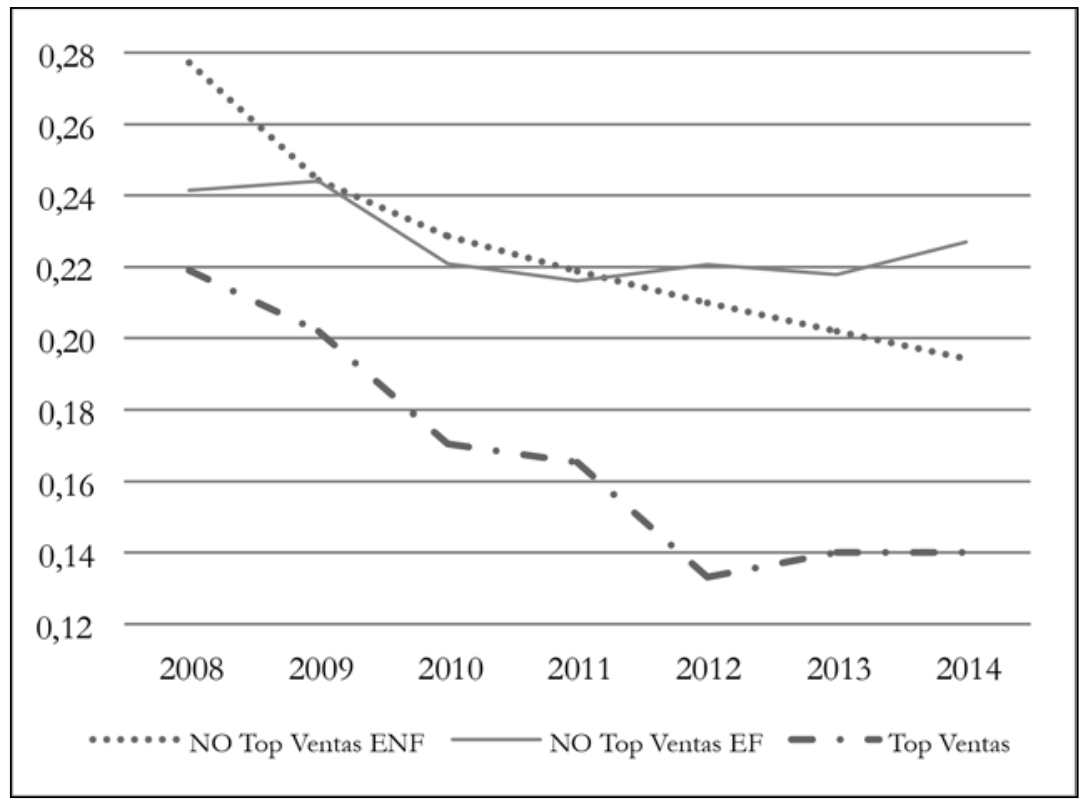

Fuente: Elaboración de los autores basado en este estudio 
El coeficiente de variación se reduce en 7.9 puntos porcentuales en las Top Ventas entre 2008 y 2014. En las ENF NO Top Ventas la reducción es de 8.3 puntos porcentuales, en tanto que en las EF NO Top Ventas la reducción es tan solo de 1.5 puntos porcentuales.

En la fila 2007-2014 de la Tabla 4 se muestran los resultados de la prueba t para la diferencia de medias y varianzas entre el 2008 y el final del estudio. Los resultados muestran que los tres grupos presentan avances significativos en el IGCCP, las que más avanzan son las ENF NO Top Ventas (5.58), seguidas de las Top Ventas (5.55), por último, las EF NO Top Ventas son las que menos avanzan (4.07). La varianza se reduce en las ENF NO Top Ventas y las NO Top Ventas, 15.81 y 19.21 unidades, respectivamente. A diferencia de las EF NO Top Ventas que se vuelven cada vez menos homogéneas, la varianza en este grupo aumenta en 6.96 unidades. Sin embargo, en ninguno de los tres casos la prueba de diferencia de varianzas es significativa, estos resultados conllevan a rechazar $\mathrm{H}_{3}$. Creemos que una clasificación de las empresas por industria hubiese sido deseable. No obstante, el tamaño de la muestra (105 empresas) no permite clasificar a las empresas por industria (serian grupos muy pequeños) donde es más probable que se presenten comportamientos isomorfos de tipo mimético, ya que la primera opción para una empresa será imitar a los líderes de su sector.

\section{Conclusiones}

Powell \& Dimaggio (1991) identifican tres mecanismos de cambio institucional isomorfo (coercitivo, mimético y normativo). Este estudio analiza la existencia de isomorfismo de forma general $\left(\mathrm{H}_{1 \mathrm{a}}\right.$ y $\left.\mathrm{H}_{1 \mathrm{~b}}\right)$ así como de los mecanismos coercitivo $\left(\mathrm{H}_{2}\right)$ y mimético $\left(\mathrm{H}_{3}\right)$ en la implementación de prácticas de gobierno corporativo durante el periodo 2008-2014 en 105 empresas inscritas en el Registro Nacional de Valores y Emisores (RNVE) de Colombia. Las hipótesis $\mathrm{H}_{1 \mathrm{a}}, \mathrm{H}_{2}$ y $\mathrm{H}_{3}$ planteadas en este estudio fueron rechazadas, por lo tanto, se concluye que las condiciones de isomorfismo propuestas por Powell \& Dimaggio (1991) no son válidas para la muestra analizada. Aunque para el caso de las ENF el com- portamiento de la dispersión es estable (se reduce periodo a periodo), estas reducciones no son significativas estadísticamente por lo que puede afirmarse que la dispersión es la misma al inicio que al final del estudio. Respecto a las EF, se encuentra que la reducción en la dispersión es diferente al encontrado en las ENF. El comportamiento de la dispersión en estas empresas es inestable (algunos años aumenta $y$ en otros se reduce), este comportamiento permitió validar $\mathrm{H}_{1 \mathrm{~b}}$ debido a que la tendencia hacia la homogeneización en la implementación de prácticas de gobierno corporativo es diferente en EF y ENF.

Consideramos que la no existencia de comportamiento isomorfo en EF y ENF se debe al poco tiempo que tiene de promulgado el Código País. Este estudio se extiende desde el 2008, que fue el año posterior al lanzamiento del código país, hasta 2014, siete años de implementación de prácticas de gobierno en Colombia. Es posible que este periodo sea corto para reflejar cambios significativos en la variación del IGCCP. Relacionado con esto, las empresas que presentan mejores estándares de gobierno corporativo aún tienen mucho espacio de mejora y lo hacen a un ritmo mayor que las empresas con menores estándares, esto hace que la diferencia se siga manteniendo entre los grupos.

El isomorfismo coercitivo resulta de presiones tanto formales como informales que sobre unas organizaciones ejercen otras de las que dependen (Powell \& Dimaggio, 1991), en este estudio encontramos que la regulación no es un factor determinante en la reducción de la dispersión en el IGCCP. Creemos que esto se debe a que, aunque existen prácticas de gobierno corporativo obligatorias, es posible que no estén bien definidas bajo los términos de Abbott \& Snidal (2000), es decir que sea una disposición vinculante, precisa y que ha sido delegada a un tercero para su interpretación y verificación del cumplimiento. Consideramos que la mayor parte de las normas de gobierno corporativo en Colombia son débiles en al menos una de estas tres dimensiones. Adicionalmente, consideramos que, debido a la falta de verificación de las entidades de supervisión, las empresas pueden manifestar que cumplen con las recomendaciones de gobierno corporativo, no obstante, es posible que en la práctica no estén im- 
plementándolas. Esta falta de supervisión hace que las presiones sean menores en las empresas que deben adoptar dichas normas y no se refleje el efecto esperado (mejoramiento constante de las prácticas de gobierno corporativo) con la regulación. Bremer, Hegazy \& Sabri (2011) sugieren que las normas profesionales (isomorfismo normativo) son complementarias, e incluso, sustitutas a la normatividad y regulación (isomorfismo coercitivo), siendo un elemento esencial en la adopción de nuevos estándares, en este sentido, futuras investigaciones deben analizar el papel que desempeñan las normas profesionales, en lugar de estudiar sólo los códigos y/o mecanismos formales de aplicación.

Respecto al isomorfismo mimético que plantea que a través de un proceso limitador se obliga a una organización en determinada población a parecerse a otras organizaciones que enfrentan las mismas condiciones ambientales (Powell \& Dimaggio, 1991), este estudio muestra que las empresas clasificadas como NO Top Ventas no tienden a parecerse a las empresas TOP Ventas. Encontramos que contrario a lo esperado, la diferencia tiende a ampliarse en el tiempo. Creemos que la clasificación por Ventas no es la más adecuada para el análisis, una clasificación de las empresas por industria hubiese sido deseable. Esta es una limitación de nuestro estudio, y se debe a que el tamaño de la muestra (105 empresas) no permite clasificar a las empresas por industria (serían grupos muy pequeños). Es probable que una clasificación de esta forma permita identificar comportamientos isomorfos de tipo mimético, ya que la primera opción para una empresa será imitar a los líderes de su sector.

Este estudio presenta múltiples implicaciones académicas y prácticas. Por un lado, los hallazgos plantean un gran reto para la academia. Es importante profundizar en la investigación en el área de la empresa familiar, empresas donde el poder y el control de la familia propietaria no siguen modas y buscan estabilizar la combinación de sus propios sistemas: el familiar, el empresarial y el patrimonial, que junto con el compromiso les otorga su principal ventaja competitiva (Gallo, 1995; Gómez-Betancourt, 2010). Este estudio mostró que este tipo de empresas presentan comportamientos diferentes como resultado de sus propias dinámicas. Es posible que las empresas familiares, como sucede en otros tipos de empresas, cuando cumplen con ciertas disposiciones de los códigos de gobierno lo hagan debido a sus propias necesidades y no por procesos de influencia relacionados con comportamientos isomorfos o miméticos (Gunathilake \& Palli, 2010). Esto sugiere que incluso, al interior de las tipologías de empresas (en este caso, familiar y no familiar) subsisten diferencias que provocan modificaciones en la implementación de prácticas de gobierno corporativo en estas empresas, un análisis a nivel de prácticas específicas de gobierno corporativo (junta directiva, asamblea de accionistas) o a nivel de industria podría validar esta afirmación. Por otro lado, se genera una gran oportunidad para analizar cualitativamente las razones por las cuales, las empresas inscritas en el RNVE no implementan de manera general las sugerencias del Código País.

Estudios previos han encontrado que el isomorfismo pierde fuerza cuando hay amplia variabilidad en los antecedentes profesionales de los CEO (Fligstein, 1993), y hechos históricos de las empresas (Ocasio, 1999), estos factores podrían aplicarse en futuros estudios que analicen el isomorfismo desde diferentes sectores económicos. En cuanto a las instituciones reguladoras, el hecho de que no exista evidencia de una tendencia hacia el isomorfismo en la adopción de prácticas gobierno corporativo puede sugerir que los esfuerzos realizados en el diseño del Código País no se verán concretados hasta que no se difundan eficazmente y hasta que las empresas no interioricen dichas prácticas, así como la influencia de las mismas en el desempeño a largo plazo. En este sentido, las instancias de control deberían generar un mecanismo de verificación para garantizar que los reportes de gobierno corporativo suministrados por las empresas reflejen su realidad empresarial.

Implicaciones prácticas para las empresas están relacionadas con la posibilidad de mejoras y seguimiento en las recomendaciones de gobierno corporativo adoptadas, es decir, que aquellos estándares que sean implementados puedan ser monitoreados y controlados periódicamente de tal forma que el comportamiento de los órganos de gobierno y sus 
responsables se modifique con el tiempo. La evidencia aquí encontrada indica que las empresas colombianas no tratan de imitar las prácticas adoptadas por empresas exitosas, esto puede deberse a: i) no las consideran como referentes, ii) perciben la adopción de prácticas de gobierno corporativo como un gasto inoficioso, o iii) las empresas están concentradas en sí mismas, es decir, en su operación y en adaptarse a la volatilidad del entorno económico y fiscal del país. En este sentido, algunos autores han señalado que las condiciones distintivas de un país a largo plazo fortalecen con el tiempo el isomorfismo local, pero lo distancia del comportamiento global (Aguilera \& Jackson, 2003). En la medida que las empresas contemplen otros beneficios del gobierno corporativo como la continuidad en el tiempo, la competitividad y la atractividad para los inversionistas, es posible que se encuentre evidencia acerca de comportamientos isomorfos en la adopción de prácticas de gobierno corporativo.

Finalmente, en cuanto a las limitaciones, en este estudio se asumió que la encuesta Código País refleja la realidad de la implementación de prácticas de gobierno corporativo, esto debido a que no existen mecanismos sólidos de verificación y las empresas pueden suministrar información no veraz con la intención de mostrar una imagen positiva y confiable al mercado. Como sugerencia para futuras investigaciones se recomienda abordar el estudio del isomorfismo desde prácticas específicas de gobierno corporativo (tamaño de la junta, independencia, número de reuniones), en este estudio se hizo desde una perspectiva general.

\section{Referencias}

Abbott, K., \& Snidal, D. (2000). Hard and soft law in international governance. International Organization, 54 (3): 421-456.

Aguilera, R. \& Jackson, G. (2003). The cross-national diversity of corporate governance: Dimensions and determinants. Academy of Management Review, 28(3), 447-465.

Barbesta, A., Giudici, G., \& Lugo, S. (2010). Mimetic isomorphism in the governance of IPO companies in Italy. Corporate Ownership and Control, 8(1A): 117-122.

Bremer, J., Hegazy, M., \& Sabri, A. (2011). Improving audit committee performance in the Middle East: do Egyptian audit profession norms support international standards? International Journal of Business Governance and Ethics, 6(3): 225-248.
Claessens, S. (2006). Corporate governance and development. The World Bank Research Observer, 21 (1): 91-122.

De Lima, L., Oliveira, M., Ponte, V. \& Rebouças, S. (2016). Práticas de governança corporativa adotadas e divulgadas pelos fundos de pensão brasileiros. Revista Contemporânea de Contabilidade, 12(27): 3-26.

Dias Filho, J., \& Moura, F. (2013). Evidenciações de Cunho Social em seis Bancos Brasileiros - Uma análise à luz da Teoria Institucional. Revista Universo Contábil, 9 (3): 6-21.

Dunne, P., \& Hughes, A. (1994). Age, size, growth and survival: UK companies in the 1980s. The Journal of Industrial Economics, XLII (2): 115-140.

Fligstein, N. (1993). The transformation of corporate control. Cambridge, MA: Harvard University Press.

Gallo, M. (1995). Empresa familiar: texto y casos. Barcelona: Estudios y Ediciones IESE.

Gómez-Betancourt, G. (2010). ¿Cómo construir un legado familiar? Un modelo para tener familias empresarias perdurables. México: Cengage Learning.

Gómez-Betancourt, G., López, V., Betancourt, R. \& Millán, P. (2012). Estudio sobre el desempeño de las empresas familiares colombianas que cotizan en la bolsa de valores, frente a las empresas no familiares. Entramado, 8(1), 28-42.

Gunathilake, W. \& Chandrakumara, P. (2010). Why do corporations accept voluntary codes on corporate governance and why is theacceptance so rapid among the corporations? A theoretical explanation. British Academy of Management (pp. 1-25). Sheffield:University of Sheffield

Holder-Webb, L., \& Cohen, J. (2012). The cut and paste society: Isomorphism in codes of ethics. Journal of Business Ethics, 107 (4): 485-509.

Lagos, C. D., \& Vecino, A. C. E. (2014). Influencia del gobierno corporativo en el costo de capital proveniente de la emisión de deuda. Estudios Gerenciales, 30(130), 73-84.

Maseda, A., Arosa, B., \& Iturralde, T. (31 de Julio de 2015). Familia empresaria y empresa familiar: estructuras de gobierno. Disponible en: http://www.ehu.eus/documents/2819611/2882007/ Familia_empresaria_y_empresa_familiar.pdf.

Meyer, J. W., \& Rowan, B. (1977). Institutionalized organizations: Formal structure as myth and ceremony. American Journal of Sociology, 83 (2): 340-363.

Moura, A., Magalhães, S., Aquino, A., \& Santos, S. (2014). Corporate Governance under the perspective of isomorphism: A BMFBOVESPA banking industry approach. Revista de Gestão, Finanças E Contabilidade, 4(3): 23-44.

Ocasio, W. (1999). Institutionalized action and corporate governance: The reliance on rules of CEO succession. Administrative Science Quarterly, 44 (2): 384-416.

Petrakis, P. (1997). Entrepreneurship and growth: creative and equilibrating events. Small Business Economics, 9 (5): 383-402.

Powell, W., \& Dimaggio, P. (1991). Retorno a la jaula de hierro: el isomorfismo institucional y la racionalidad colectiva en los campos organizacionales. En: W. Powell y P. Dimaggio (Eds.), El nuevo institucionalismo en el análisis organizacional. México: Fondo de Cultura Económica. 
Riaz, Z., Ray, S., Ray, P. K., \& Kumar, V. (2015). Disclosure practices of foreign and domestic firms in Australia. Journal of World Business, 50 (4): 781-792.

San Martin-Reyna, J., \& Duran-Encalada, J. (2012). The relationship among family business, corporate governance and firm performance: Evidence from the Mexican stock exchange. Journal of Family Business Strategy, 3(2): 106-117.

Suess, J. (2014). Family governance-Literature review and the development of a conceptual model. Journal of Family Business Strategy, 5 (2): 138-155.

Superintendencia de Valores. (2001). Resolución 275 de 2001. Disponible en: https://www.superfinanciera.gov.co/jsp/loader. jsf?IServicio $=$ Publicaciones\&ITipo $=$ publicaciones\&lFuncion $=$ loadContenidoPublicacion\&id $=821 \& \mathrm{dPrint}=1$
Superintendencia Financiera de Colombia (2007). Código de mejores prácticas corporativas. Disponible en: https:// www.superfinanciera.gov.co/SFCant/Codigopais/textos/ codigopias.pdf.

Thorsell, A., \& Cornelius, B. (2009). Coercion, Copy-Cats, and Colleagues: Staffing the Board of the IPO Company. Corporate Ownership and Control, 7 (1): p.105.

Whetten, D. (1987). Organizational growth and decline processes. Annual Review of Sociology, 13: 335-358.

Zakaria, I. (2012). Performance measures, benchmarks and targets in executive remuneration contracts of UK firms. The British Accounting Review, 44 (3): 189-203. 